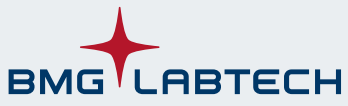

The Microplate Reader Company

\title{
Real-time monitoring of cellular metabolic activity: intracellular oxygen
}

\section{Despite the important metabolic information provided by intracellular oxygen contents, most studies omit measurement or regulation of oxygen. The CLARIOstar ${ }^{\circledR}$ microplate reader from BMG LABTECH enables researchers to use oxygen-sensitive probes to obtain a more complete picture of cellular activity.}

Intracellular oxygen content reflects a cell's metabolic activity and depends on surrounding oxygen levels. However, the majority of cellular studies use ambient oxygen $\left(\mathrm{O}_{2}\right)$ and neglect to consider oxygen gradients between atmosphere and medium or between medium and intracellular compartment. In order to relate levels of cellular oxygenation to cellular behavior, Luxcel Biosciences developed oxygen-sensitive probes.

The CLARIOstar ${ }^{\circledR}$ microplate reader reliably detects these oxygensensitive probes. With the use of its temperature control options and atmospheric control unit (ACU), both optimal physiological conditions and hypoxic conditions can be reproduced, and the effect of these states on intracellular oxygen can be monitored (Fig. 1).

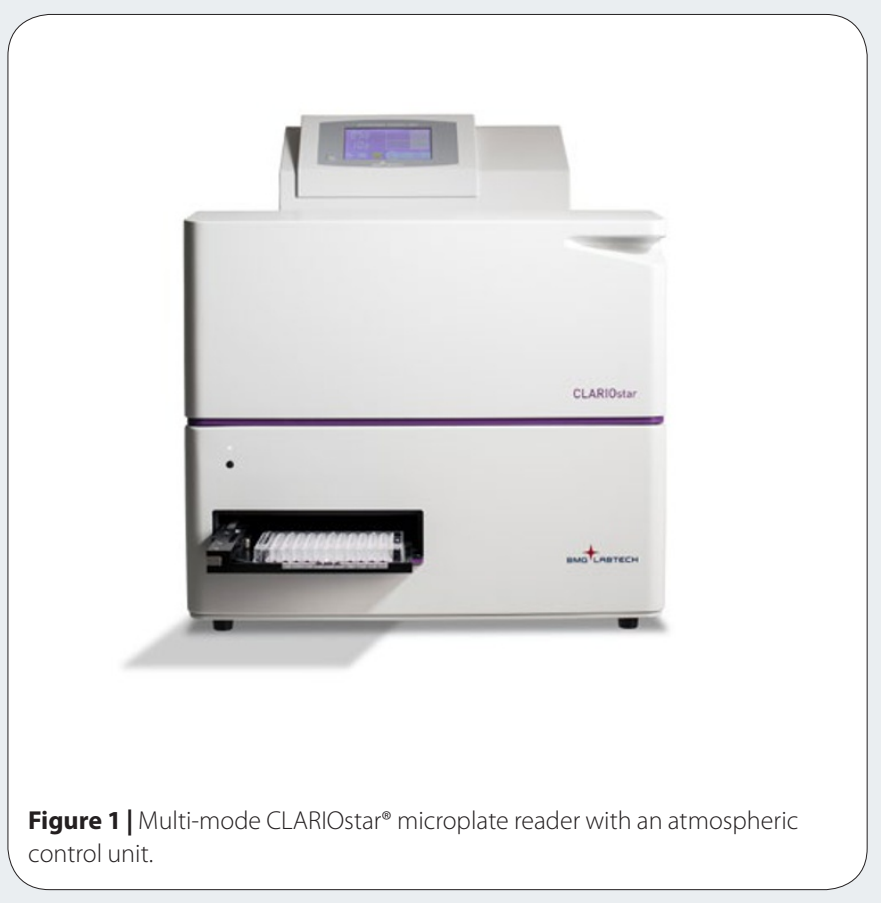

Andrea Krumm ${ }^{1} \&$ Conn Carey ${ }^{2}$

${ }^{1}$ BMG LABTECH, Ortenberg, Germany. ${ }^{2}$ Luxcel Biosciences, Cork, Ireland. Correspondence should be addressed to A.K. (andrea.krumm@bmglabtech.com).
Why should intracellular oxygen be monitored?

Molecular oxygen is used as an oxidizing agent in cellular respiration. Thus, intracellular oxygen is an established marker for the state of cellular metabolism. Furthermore, changes in amounts of intracellular oxygen occur in the course of pathologies, such as in cancerous cells, which typically show reduced oxidative phosphorylation. Analysis of the intracellular oxygen content over time tracks the effect of drug treatment on cell metabolism and reveals metabolic changes that are related to disease states. The intracellular oxygen content depends not only on the cell's state but also on environmental oxygen. Therefore, it is necessary for cellular oxygen measurements to keep the composition of the surrounding oxygen atmosphere stable, a fact that is often ignored in cellular studies.

How can $\mathrm{O}_{2}$ be monitored in cells?

The MitoXpress ${ }^{\circledR}$ Intra Intracellular Oxygen Assay by Luxcel Biosciences measures intracellular oxygen concentrations $\left(\left[\mathrm{O}_{2}\right]\right)$ in both 2D and 3D culture systems. The oxygen-sensitive probe is taken up by the cells during an overnight loading period and responds in real time to any changes in intracellular $\left[\mathrm{O}_{2}\right]$. The probe emits varying levels of phosphorescence depending on the $\left[\mathrm{O}_{2}\right]$. A high amount of available oxygen quenches the signal, leading to a low phosphorescence value. As cells respire, the $\left[\mathrm{O}_{2}\right]$ will decrease, leading to an increase in phosphorescence signal.

What is needed for intracellular $\mathrm{O}_{2}$ monitoring?

- MitoXpress ${ }^{\circledR}$ Intra (MX-300, http://www.luxcel.com)

- CLARIOstar ${ }^{\circledR}$ microplate reader equipped with an ACU

- Cellular sample (2D or 3D cell culture, such as HepG2 cells)

\section{Data calculation}

A data transform by the MARS software is required to convert signal to lifetime and lifetime to $\% \mathrm{O}_{2}$. The $\mathrm{BMG} \mathrm{LABTECH}$ readers, including the CLARIOstar ${ }^{\circledR}$, come with pre-installed measurement protocols and an $\mathrm{O}_{2}$ scale that allow the user to undertake data conversion with a single mouse click. 
Cellular response to decreased ambient $\mathrm{O}_{2}$

The sample data presented in Figure 2 illustrate the effect of cell respiration on the $\left[\mathrm{O}_{2}\right]$ experienced by liver cells grown in a $3 \mathrm{D}$ collagen culture (RAFT TM). The CLARIOstar ${ }^{\circledR}$ ACU was used to create a stepwise series of atmospheric environments between $19 \%$ and close to $0 \%$ oxygen.

The initial $\left[\mathrm{O}_{2}\right]$ set by the microplate reader ACU was $~ 19 \%$; however, the measured $\left[\mathrm{O}_{2}\right]$ of cells in the $3 \mathrm{D}$ culture was around $10 \%$. This decrease in $\left[\mathrm{O}_{2}\right]$ was due to rapid local depletion of oxygen, which is consumed through cellular respiration by this metabolically active cell type. At each environmental $\left[\mathrm{O}_{2}\right]$ value set, the actual $\left[\mathrm{O}_{2}\right]$ experienced by these liver cells was between $2 \%$ and $10 \%$ lower. The graph shows that at the levels of environmental oxygen typically used by researchers studying hypoxia $(\sim 5 \%)$, the local $\left[\mathrm{O}_{2}\right]$ of the cells was close to zero.

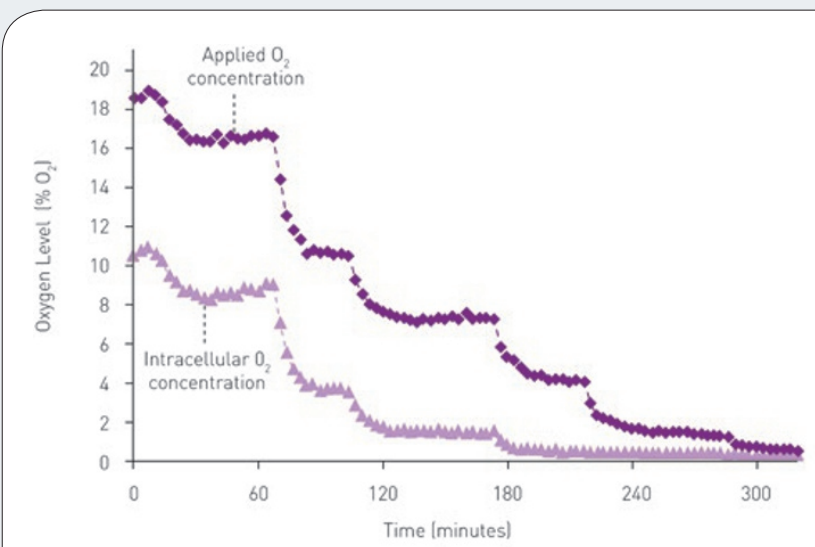

Figure $\mathbf{2}$ | Observed intracellular $\mathrm{O}_{2}$ concentrations (triangles) in samples containing 3D HepG2 cells in response to decreasing atmospheric $\mathrm{O}_{2}$ conditions (diamonds) realized by the CLARIOstar ${ }^{\circledR}$ ACU.

\section{Effect of compound addition}

The sample data in Figure 3 illustrate the immediate and longer-term effects of drugs that affect mitochondrial function and cell metabolism on cells in 2D (Fig. 3a) and 3D (Fig. 3b) culture. The capability to allow sequential compound additions is a feature of the CLARIOstar ${ }^{(\mathbb{R} /} \mathrm{s}$ onboard injectors, making it possible to study opposing effects of different compounds on cellular $\left[\mathrm{O}_{2}\right]$ in the same well.

Cell respiration reduced the $\left[\mathrm{O}_{2}\right]$ from ambient levels to a baseline of $\sim 10 \%$ for cells grown as a monolayer and to $\sim 9 \%$ for cells grown in $3 \mathrm{D}$ culture. Increasing the oxygen consumption rate through treatment with the mitochondrial uncoupler FCCP caused an acute and dramatic decrease in $\left[\mathrm{O}_{2}\right]$ to $\sim 2-3 \%$, whereas complete inhibition of respiration by the mitochondrial inhibitor antimycin returned $\left[\mathrm{O}_{2}\right]$ to ambient levels. DMSO addition served as control and had no significant influence on oxygen concentration.

Physiological $\mathrm{O}_{2}$ use and the substrate environment in which cells are cultured significantly affect signal transduction and cellular response to drug treatment. Luxcel's MitoXpress ${ }^{\circledR}$ Intra Intracellular Oxygen Assay kit provides researchers with an easy-to-use tool with which they can measure the actual $\left[\mathrm{O}_{2}\right]$ of their cell culture system to achieve greater understanding of effects on cellular physiology. a

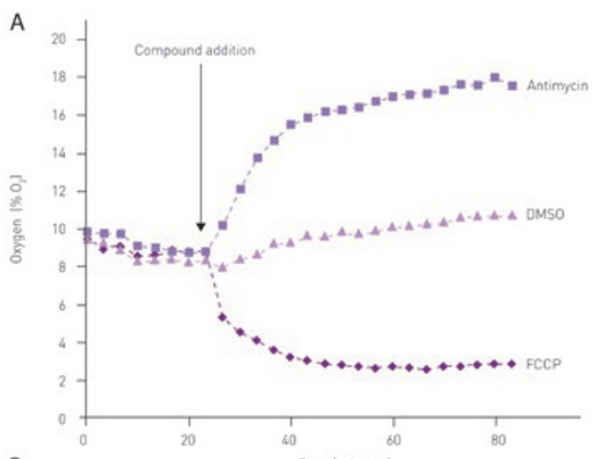

b

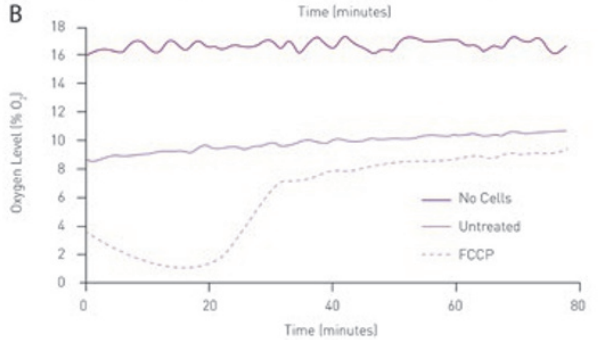

Figure $\mathbf{3}$ | Intracellular oxygen content in response to mitochondria-targeting drugs. (a) Observed intracellular $\mathrm{O}_{2}$ concentrations in a fully confluent monolayer of HepG2 cells (compounds added using the reader's onboard injectors). (b) The effect of drug treatment on cellular oxygenation in HepG2 3D RAFT ${ }^{\mathrm{M}}$ structures. Results were obtained using MitoXpress ${ }^{\oplus}$ Intra at 21\% applied oxygen.

\section{Conclusion}

The CLARIOstar ${ }^{\circledR}$ equipped with an ACU is an excellent choice for measuring intracellular $\left[\mathrm{O}_{2}\right]$. The instrument is equipped with temperature and atmospheric controls as well as shaking and injection options for all requirements. These features are necessary for long-term cell culture while measuring alterations in cell metabolism caused by, for example, a decreased ambient oxygen content. The reader's onboard injectors can be used to add compounds.
This article was submitted to Nature Methods by a commercial organization and has not been peer reviewed. Nature Methods takes no responsibility for the accuracy or otherwise of the information provided. 